\title{
Beamforming of Smart Antenna Using a Combined Tchebyscheff Distribution and Constant Modulus Algorithm
}

\author{
Subhashree Nibedita Baliarsingh, Anupama Senapati, Arindam Deb, Jibendu Sekhar Roy \\ School of Electronics Engineering, KIIT University, Bhubaneswar 751024, Odisha, India \\ subhashreeb4@gmail.com, senapati.anupama@gmail.com, adebfet@kiit.ac.in,drjsroy@rediffmail.com
}

\begin{abstract}
The objective of this paper is to improve the performance of adaptive smart antenna using a hybrid algorithm combining constant modulus algorithm (CMA) and array synthesis method. Adaptive beamforming using Tchebyscheff distribution, an array synthesis method, along with constant modulus algorithm (TDCMA) is compared with the performances of beamforming using constant modulus algorithm only. Reduction of side lobe level is an important task to minimize unwanted interference for other users in mobile network. Side lobe level of about $9 \mathrm{~dB}$ lower than CMA algorithm is achieved using TDCMA algorithm. Investigations, presented here, may be useful for mobile network.
\end{abstract}

Keyword-Adaptive beamforming, Constant modulus algorithm, Tchebyscheff distribution, Side lobe level reduction

\section{INTRODUCTION}

In mobile communication, network performance can be improved by using adaptive smart antenna. Smart antenna is an antenna array with digital signal processing unit which after estimating direction of arrival (DOA), generates radiation beam along the desired user and produces null towards the undesired interferer [1-3]. Appreciable power saving is possible using smart antenna in mobile network in addition to enhancement of signal quality, network capacity and coverage area [4].

In smart antenna, the received signals at the different antennas are multiplied with complex weights and then adaptively weights are summed up [4-5]. Various types of beamforming algorithms, having their advantages and disadvantages are available [3-8]. In addition to various methods of DOA estimations, many iterative schemes applicable to adaptive beamforming have been described [3]. A sequential quadratic programming based algorithm is used for multi-lobe pattern and for adaptive nulling of the pattern [5]. A complex quaternion least mean square (LMS) algorithm is used [6] for beamforming of polarization-sensitive electromagnetic vectorsensor. An adaptive algorithm to control main beam and side lobe of adaptive array, based on amplitude approaching algorithm, is reported [7]. Numerical and experimental results for an eight elements linear smart antenna array using a set of electronically driven vector modulators, are described in [8]. To improve the performance of adaptive smart antenna, array synthesis methods are used in [9-10]. Genetic algorithm is coupled with the Schelkunoff synthesis method for uniform linear and planar array design [9]. Tchebyscheff distribution (TD) is used with beamforming constraint stability least mean square (CSLMS) algorithm for adaptive antenna [10].

In this paper, array synthesis method, Tchebyscheff distribution (TD) for current amplitude distribution in linear antenna array of 16 antenna elements is used with constant modulus algorithm (CMA) [11-12]. First, CMA algorithm is used for beam formation for adaptive smart antenna and then the program is modified to incorporate Tchebyscheff distribution along with CMA algorithm (TDCMA). Side lobes are causes of interferences for other users in a mobile cell. Attention is given to reduce side lobe level using the new proposed algorithm.

\section{AdAPtive BeAmforming of SMART AnTenna Using CMA AND TDCMA}

Constant modulus algorithm (CMA) exploits the constant modularity of the signal to adapt the parameters [11-12].The algorithm is best used with signals that have a constant envelope. If $x(k)$ is the input signal, then the output signal $y(k)$ is given by [11-12]

$$
y(k)=w_{C M A}^{H}(k) x(k)
$$

The weight $(w)$ updating equation is similar to LMS algorithm, but the error is computed from the actual received signal, not from a training sequence. Weight updating equation is given by [11-12]

$$
w_{C M A}(k+1)=w_{C M A}(k)-\mu x(k)\left(|y(k)|^{2}-1\right) y^{H}(k)
$$

Where $\mathrm{H}$ denotes the transpose conjugate and $\mu$ is the step size parameter. 
A uniform linear array of inter-element spacing 'd' is shown in Fig 1.

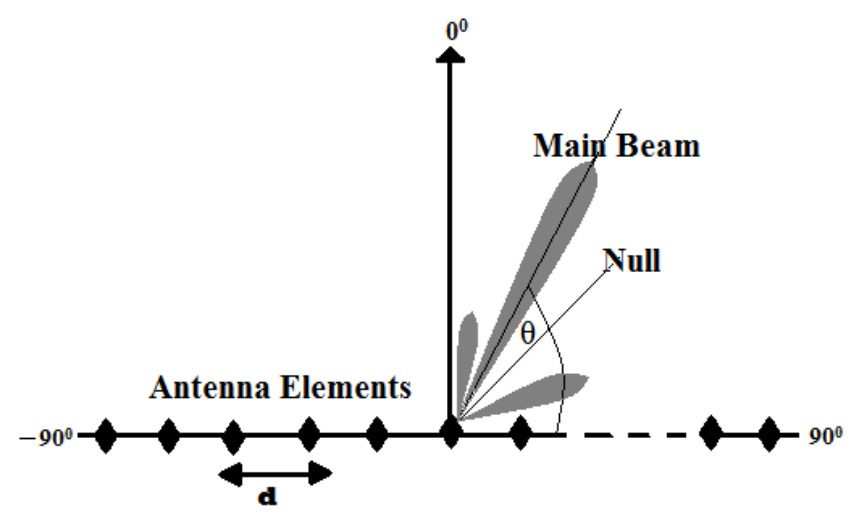

Fig. 1. Linear antenna array configuration

For a main beam at an angle $\theta$, the array factor, is expressed as [13-14]

$$
A F_{L}=\sum_{n=1}^{N} A_{n} e^{j(n-1)(\beta d \cos \theta+\alpha)}
$$

Where phase factor $\beta=2 \pi / \lambda$ and to generate the main beam at wavelength $\lambda$ toward the desired beam direction $\theta^{0}$ from the broadside direction, the progressive phase shift is

$$
\alpha=\frac{-2 \pi d}{\lambda} \cos \theta
$$

Tchebyscheff distribution (TD) for current amplitude [13-14], fed in antenna, is used for antenna array synthesis to achieve narrowest beam for a given side lobe level. Also it is used to obtain lowest side lobe level for a given beam width. Tchebyscheff distribution, expressed in polynomial form as [13-14]

$$
\begin{aligned}
& T_{m}(x)=\left\{\begin{array}{cc}
\cos \left(m \cos ^{-1} x\right) ; & -1<x<+1 \\
\cosh \left(m \cosh ^{-1} x\right) ; & |x|>+1
\end{array}\right. \\
& T_{m}\left(x_{o}\right)=b
\end{aligned}
$$

It can be calculated [12] that if, $b=\cosh \rho$, then

$$
x_{o}=\cosh (\rho / m)
$$

Beamwidth is directly related to ' $b$ ' and ' $x_{o}$ ' is related to the position of main beam.

In this paper, in adaptive beam formation, array synthesis method, Tchebyscheff distribution is used along with beamforming algorithm CMA to generate adaptive beam in a particular direction as well as in the direction of pre-defined null. The cost function is the final array factor for Tchebyscheff array, is given by

$$
A F=A F_{L} T_{m}(x)
$$

Normalized array factor is

$$
A F_{\text {norm }}=\frac{A F}{A F_{\text {max }}}
$$

In simulation, particular directions of main beam and null are given as input for 16 elements linear antenna array. The value of inter-element spacing ' $d$ ' is $0.5 \lambda$. Then in Tchebyscheff distribution side lobe level is fixed at $-25 \mathrm{~dB}$. In the TDCMA algorithm, first the current amplitudes, fed to the antennas, are determined using Tchebyscheff distribution for a particular value of SLL. Then CMA algorithm is used for weight updating to generate beam and null in desired directions. Normalized array factors of adaptive array for beam direction $15^{\circ}$ and null direction $22^{\circ}$, using CMA and TDCMA, are compared in Fig 2. 


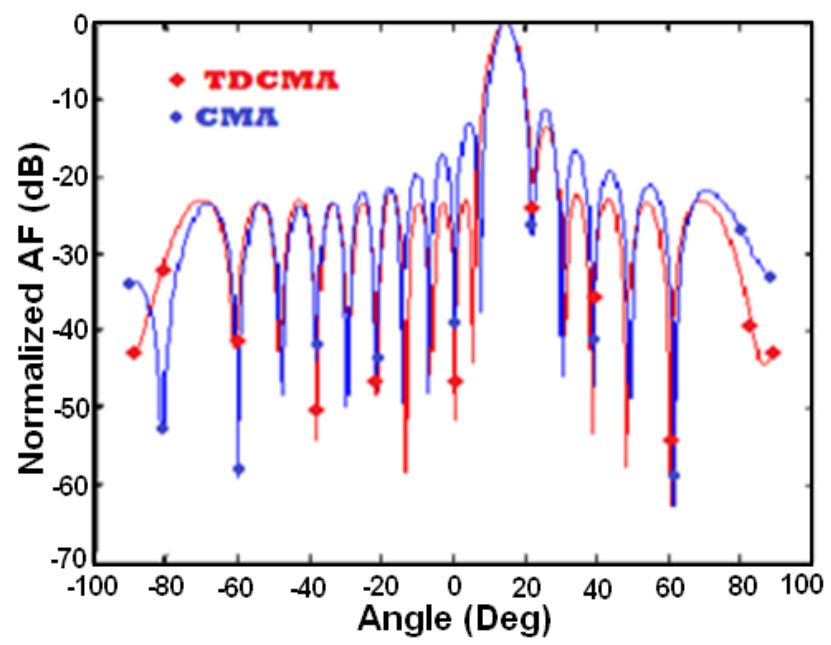

Fig. 2. Array pattern at beam direction $15^{\circ}$ and null direction $22^{\circ}$

Similarly, normalized array factor of adaptive array for beam direction $35^{\circ}$ and null direction $45^{\circ}$ using CMA and TDCMA is compared in Fig 3 and normalized array factor of adaptive array for beam direction $10^{\circ}$ and null direction $35^{0}$ using CMA and TDCMA is compared in Fig 4. Here, in CMA, step size, $\mu=0.003$.

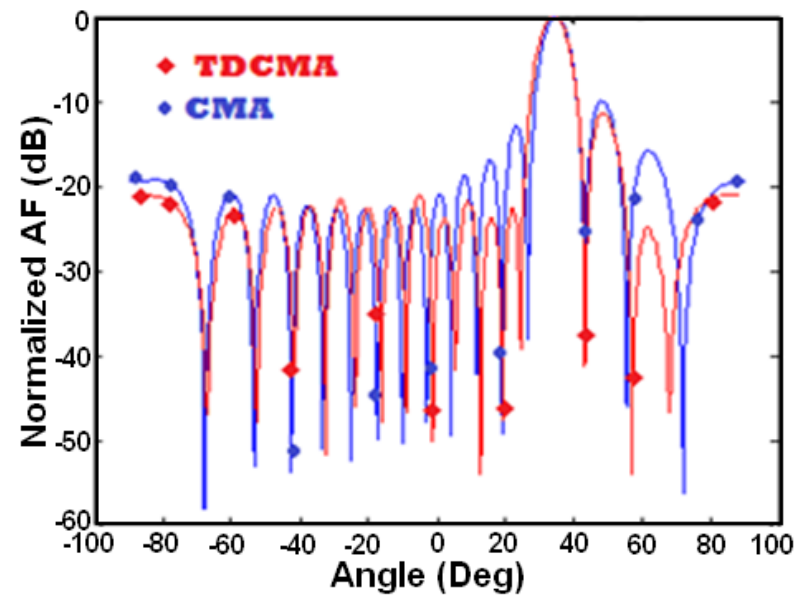

Fig. 3. Array pattern at beam direction $35^{\circ}$ and null direction $45^{\circ}$

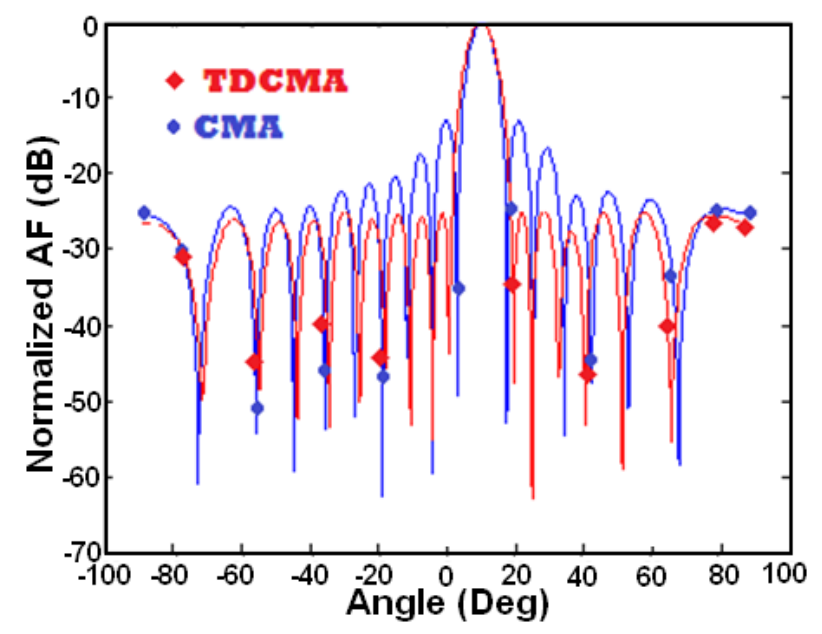

Fig. 4. Array pattern at beam direction $10^{\circ}$ and null direction $35^{\circ}$

The summary of results with respect to null direction, null-to-null beamwidth (BW) and side lobe level (SLL), is tabulated in Table I. In all the algorithms, desired main beam directions achieved perfectly, whereas, in some cases there is small shift of null directions from the desired value. 
TABLE I PERFORMANCE OF BEAMFORMING USING CMA AND TDCMA ALGORITHMS

\begin{tabular}{|c|c|c|c|c|c|c|}
\hline & \multicolumn{3}{|c|}{ Beamforming using CMA } & \multicolumn{3}{c|}{ Beamforming using TDCMA } \\
\hline $\begin{array}{c}\text { Desired beam \& null } \\
\text { directions }\end{array}$ & $\begin{array}{c}\text { Beam } \\
\text { direction }\end{array}$ & $\begin{array}{c}\text { Null } \\
\text { direction }\end{array}$ & $\begin{array}{c}\text { Maximu } \\
\text { m SLL }\end{array}$ & $\begin{array}{c}\text { Beam } \\
\text { direction }\end{array}$ & $\begin{array}{c}\text { Null } \\
\text { direction }\end{array}$ & $\begin{array}{c}\text { Maximu } \\
\text { m SLL }\end{array}$ \\
\hline Beam at $15^{0}$, null at $22^{0}$ & $15^{0}$ & $22^{0}$ & $-10.5 \mathrm{~dB}$ & $15^{0}$ & $22.5^{0}$ & $-13.5 \mathrm{~dB}$ \\
\hline Beam at $35^{0}$, null at $45^{0}$ & $35^{0}$ & $44.5^{0}$ & $-9.0 \mathrm{~dB}$ & $35^{0}$ & $44.5^{0}$ & $-12.5 \mathrm{~dB}$ \\
\hline Beam at $10^{0}$ and null at $35^{0}$ & $10^{0}$ & $34^{0}$ & $-12.5 \mathrm{~dB}$ & $10^{0}$ & $34.5^{0}$ & $-21.5 \mathrm{~dB}$ \\
\hline
\end{tabular}

Using TDCMA algorithm, for desired beam direction $15^{\circ}$, null direction $22^{0}$, side lobe level reduction of 3 $\mathrm{dB}$, for desired beam direction $35^{\circ}$, null direction $45^{\circ}$, side lobe level reduction of $3.5 \mathrm{~dB}$ and for desired beam direction $10^{\circ}$, null direction $35^{\circ}$, side lobe level reduction of $9 \mathrm{~dB}$, are achieved compared to CMA algorithm.

\section{III.DISCUSSION AND CONCLUSION}

A new method of adaptive beamforming for smart antenna using Tchebyscheff distribution (TDCMA) and CMA algorithm is presented in this short communication. Lower side lobe level is achieved using TDCMA compared to using only CMA algorithm. Number of iterations in each computation is 100 . Simulations are done using large number of step-size values in CMA algorithm. The step-size values $(\mu)$, in all the cases used here, are the values for which best results (with respect to beam direction, null direction, SLL) are found. In array synthesis using Tchebyscheff distribution (TD), the number of nulls $(\mathrm{m})$ is decided by the polynomial $\mathrm{T}_{\mathrm{m}}(\mathrm{x})$. But in adaptive beam formation when TD is used with CMA algorithm, the number of nulls may not be same as expected using TD only. In all the cases of TDCMA, the directions of main beams are achieved accurately. Using TDCMA, the directions of nulls are shifted slightly from the desired directions. In those cases sharp nulls are not produced at the desired null directions but the levels of radiation in desired null directions are far below the effective levels $(-20 \mathrm{~dB})$.

\section{REFERENCES}

[1] T. K. Sarkar, M. C. Wicks, M. Salazar-Palma and Robert J. Bonneau, Smart Antennas, Wiley-IEEE Press, USA, 2003.

[2] F. Gross, Smart antenna for wireless communication, McGraw-Hill, USA, 2005.

[3] S. Bellofiore, C. A. Balanis, J. Foutz and A. S. Spanias, "Smart-antenna systems for mobile communication network-part 1, overview and antenna design," IEEE Antennas and Propagation Magazine, vol. 44, pp. 145- 154, 2002.

[4] L. C. Godara, "Application of antenna arrays to mobile communications-part II: beam-forming and direction-of-arrival considerations," Proceedings of the IEEE, vol. 85, pp. 1195-1245, 1997.

[5] M. Mouhamadou, P. Vaudon and M. Rammal, "Smart antenna array patterns synthesis: null steering and multi-user beamforming by phase control," Progress In Electromagnetics Research, vol. 60, pp.95-106, 2006.

[6] J-W Tao and W-X Chang, "Adaptive beamforming based on complex quaternion processes," Mathematical Problems in Engineering, vol. 2014, pp. 1-10, 2014

[7] J. Li, R. Jin and Y. Sheng, "A fast synthesis algorithm of adaptive beams for smart antennas," Microwave Opt Technol Lett., vol. 36, pp. 503-507, 2003.

[8] R. Azaro, L. Ioriatti, M. Martinelli, M. Benedetti and A. Massa, "An experimental realization of a fully adaptive smart antenna," Microwave Opt Technol Lett., vol. 50, pp. 1715-1716, 2008.

[9] A. Recioui and A. Azrar, "Use of genetic algorithms in linear and planar antenna array synthesis based on Schelkunoff method," Microwave Opt Technol Lett., vol. 49, pp. 1619-1623, 2007.

[10] S. N. Baliarsingh, A. Senapati, A. Deb and J. S. Roy, "Adaptive beam formation in smart antenna - a new combined method using Tchebyscheff distribution and constrained stability least mean square algorithm," Asian Journal of Applied Sciences, vol. 4, pp. 379386, 2016.

[11] A-J. Van Der Veen and A. Paulraj, “An analytical constant modulus algorithm," IEEE Transactions on Signal Processing, vol.44, pp. $1-19,1996$.

[12] R. Divya and T. Jayasimha, "Adaptive beamforming algorithm for smart WiMAX antenna," International Journal of Advance Research in Computer Science and Management Studies, vol. 2, pp. 20-29, 2014.

[13] C. A. Balanis, Antenna Theory - Analysis and Design, $3^{\text {rd }}$ Ed., Wiley, USA, 2005.

[14] E. C. Jordan and K. G. Balmain, Electromagnetic Waves and Radiating Systems, $2^{\text {nd }}$ Ed., Prentice-H all of India, New Delhi, 2009.

\section{AUTHOR PROFILE}

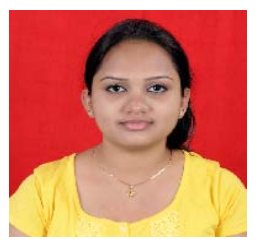

Subhashree Nibedita Baliarsingh is an assistant professor in the Electronics and Communication Engineering department, Mahavir Institute of Engineering and Technology, Bhubaneswar, Odisha, India. Earlier, she received her B. Tech. degree from ITER, SOA University, Bhubaneswar in 2013 and M. Tech. from KIIT University, Bhubaneswar in 2016. 

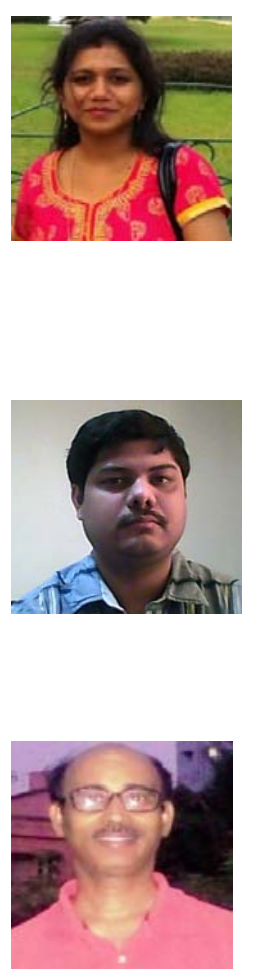

Anupama Senapati is an assistant professor in the School of Electronics Engineering, KIIT University, Bhubaneswar, India. Earlier, she received her B. Tech. degree from College of Engineering Bhubaneswar (CEB), Bhubaneswar in 2010 and M. Tech. from KIIT University, Bhubaneswar in 2012. She has received her Ph. D. degree from KIIT University, Bhubaneswar in 2016. Her areas of research work are antennas, antenna arrays and smart antenna. She has published about 20 papers in international journals and conferences

Arindam Deb is an assistant professor in the School of Electronics Engineering, KIIT University, Bhubaneswar, India. Earlier, he received M. Tech. degree from ETCE department, Jadavpur University, Kolkata, India in 2008. He has received his Ph. D. degree in 2017 from Jadavpur University, Kolkata. His area of research interest is applications of evolutionary algorithms in antenna and antenna array design. He has published more than 20 papers in international journals and conferences.

Jibendu Sekhar Roy is a professor in the School of Electronics Engineering, KIIT University, Bhubaneswar, Odisha, India. From 1998 to 2009, he was lecturer, reader and professor in the ECE department of Birla Institute of Technology, Mesra, Ranchi, India. $\mathrm{He}$ has received Ph. D. degree from the Department of Electronics \& Telecommunication Engineering, Jadavpur University, Calcutta, India in 1991. From 1991 to 1993, he was a post-doctoral research associate of CNRS, Govt. of France in IRCOM, University of Limoges, France. From 1994 to 1998, he was a research associate of CSIR in ETCE department, Jadavpur University, Calcutta. His research interest includes microwave and millimeterwave antennas for wireless communication, optimization of thinned array antenna, smart antennas, channel assignment \& MIMOOFDM algorithms, cognitive radio. Dr. Roy has published about 200 papers in international journals and symposia. He has presented Invited lectures/research papers in workshops and symposia in various Institutes in India and other countries, like, France, USA, Indonesia, Italy, Denmark, Czech Republic and South Korea. The name of Dr JS Roy has been listed in 2012 Marquis World Who's Who. 